\title{
Bunched soliton states in weakly coupled sine-Gordon systems
}

\author{
Grønbech-Jensen, N.; Samuelsen, Mogens Rugholm; Lomdahl, P. S.; Blackburn, J. A.
}

Published in:

Physical Review B

Link to article, DOI:

10.1103/PhysRevB.42.3976

Publication date:

1990

Document Version

Publisher's PDF, also known as Version of record

Link back to DTU Orbit

Citation (APA):

Grønbech-Jensen, N., Samuelsen, M. R., Lomdahl, P. S., \& Blackburn, J. A. (1990). Bunched soliton states in weakly coupled sine-Gordon systems. Physical Review B, 42(7), 3976-3980.

https://doi.org/10.1103/PhysRevB.42.3976

\section{General rights}

Copyright and moral rights for the publications made accessible in the public portal are retained by the authors and/or other copyright owners and it is a condition of accessing publications that users recognise and abide by the legal requirements associated with these rights.

- Users may download and print one copy of any publication from the public portal for the purpose of private study or research.

- You may not further distribute the material or use it for any profit-making activity or commercial gain

- You may freely distribute the URL identifying the publication in the public portal

If you believe that this document breaches copyright please contact us providing details, and we will remove access to the work immediately and investigate your claim 


\title{
Bunched soliton states in weakly coupled sine-Gordon systems
}

\author{
N. Grønbech-Jensen and M. R. Samuelsen \\ Physics Laboratory I, The Technical University of Denmark, DK-2800 Lyngby, Denmark \\ P. S. Lomdahl \\ Theoretical Division, Los Alamos National Laboratory, Los Alamos, New Mexico 87545
}

\section{J. A. Blackburn}

Department of Physics and Computing, Wilfrid Laurier University, Waterloo, Ontario, Canada N2L $3 C 5$

(Received 5 February 1990; revised manuscript received 25 April 1990)

\begin{abstract}
The interaction between solitons of two weakly coupled sine-Gordon systems is considered. In particular, the stability of bunched states is investigated, and perturbation results are compared with numerical results.
\end{abstract}

\section{INTRODUCTION}

Recently there has been much interest in the subject of coupled and perturbed sine-Gordon systems. ${ }^{1-4}$ The investigation of the sine-Gordon system, especially, has been done to model the fluxon dynamics of long Josephson junctions (see, e.g., Refs. 5-7), where a magnetic flux quantum is modeled by a localized solitary $2 \pi$-kink solution of the perturbed sine-Gordon equation (PSGE) given by

$$
\phi_{x x}-\phi_{t t}-\sin \phi=\alpha \phi_{t}-\beta \phi_{x x t}-\eta \text {. }
$$

Here $\phi$ is the phase difference between the quantummechanical wave functions of the two separated superconductors. The spatial dimension $(x)$ is normalized to the Josephson penetration depth $\lambda_{J}$ and the time dimension $(t)$ to the inverse plasma-resonance frequency $\omega_{0}^{-1}$. The normalized current density of Cooper pairs tunneling through the junction is represented by the $\sin \phi$ term and the density of tunneling quasiparticles is represented by the $\alpha \phi_{t}$ term, which is a dissipative term in the equation. Surface loss in the superconductors is represented by the parameter $\beta$. The power input is $\eta$ representing the applied bias current density forced through the junction. In this paper we will study the dynamics of the interaction between two solitons of different but weakly coupled sine-Gordon systems (SGS's). As shown in Refs. 1 and 2, two colliding solitons of different SGS's, can form a bunched state, due to dissipation. Using the adiabatic perturbation method from Ref. 5, simple criteria for the existence of bunched states can be obtained and good agreement between perturbation results and numerical simulations of the perturbed and coupled wave equations is found in the appropriate limit. Two kinds of energy conserving coupling mechanisms will be discussed in detail (inductive and capactive). In particular, the inductively coupled sine-Gordon systems are of interest, since good agreement between experiments and numerical simulations is found ${ }^{3}$ when inductively coupled SGS's are used to model the phase locking of fluxons in a system of two parallel long Josephson junctions (LJJ's).

\section{THEORY}

Considering the Lagrangian density $L$ of two coupled one-dimensional sine-Gordon systems of the two variables $\phi$ and $\psi$ :

$$
\begin{aligned}
L= & \frac{1}{2}\left(\phi_{t}^{2}-\phi_{x}^{2}+\psi_{t}^{2}-\psi_{x}^{2}\right) \\
& -2+\cos \phi+\cos \psi+\Delta_{1} \phi_{x} \psi_{x}+\Delta_{2} \phi_{t} \psi_{t} .
\end{aligned}
$$

The equations of motion for $\phi$ and $\psi$ are then the two coupled sine-Gordon equations, where the coupling terms are nondissipative, $\Delta_{1}$ and $\Delta_{2}$ being the coupling constants,

$$
\begin{aligned}
& \phi_{x x}-\phi_{t t}-\sin \phi=\Delta_{1} \psi_{x x}+\Delta_{2} \psi_{t t}, \\
& \psi_{x x}-\psi_{t t}-\sin \psi=\Delta_{1} \phi_{x x}+\Delta_{2} \phi_{t t} .
\end{aligned}
$$

From Eq. (2) we also get the total energy $H$ of the system as

$$
\begin{aligned}
& H=H_{0}+H_{I}, \\
& H_{0}=\int_{-\infty}^{\infty}\left(\frac{1}{2} \phi_{t}^{2}+\frac{1}{2} \phi_{x}^{2}+\frac{1}{2} \psi_{t}^{2}+\frac{1}{2} \psi_{x}^{2}\right. \\
& \quad+2-\cos \phi-\cos \psi) d x, \\
& H_{I}=\int_{-\infty}^{\infty}\left(\Delta_{2} \phi_{t} \psi_{t}-\Delta_{1} \phi_{x} \psi_{x}\right) d x,
\end{aligned}
$$

where we will denote $H_{I}$ for the interaction energy, since $H_{0}$ is the expression for the total energy of the two sineGordon systems with no coupling.

The coupled sine-Gordon systems, can be modeled as shown in Fig. 1, where two discrete analogs of LJJ's are inductively and capacitively coupled. The LJJ's are represented by the critical current density $I_{c}$, the shunt capacitance per unit length $C_{1}$, the leakage conductance per unit length $G_{\alpha}$, the surface resistance per unit length $R_{\beta}$, and the surface inductance per unit length $L$. The bias current densities of the two LJJ's are $I_{b 1}$ and $I_{b 2}$, respectively. The coupling of the LJJ's is given by the capacitance per unit length $C_{2}$ and the mutual inductance per unit length $M$. 


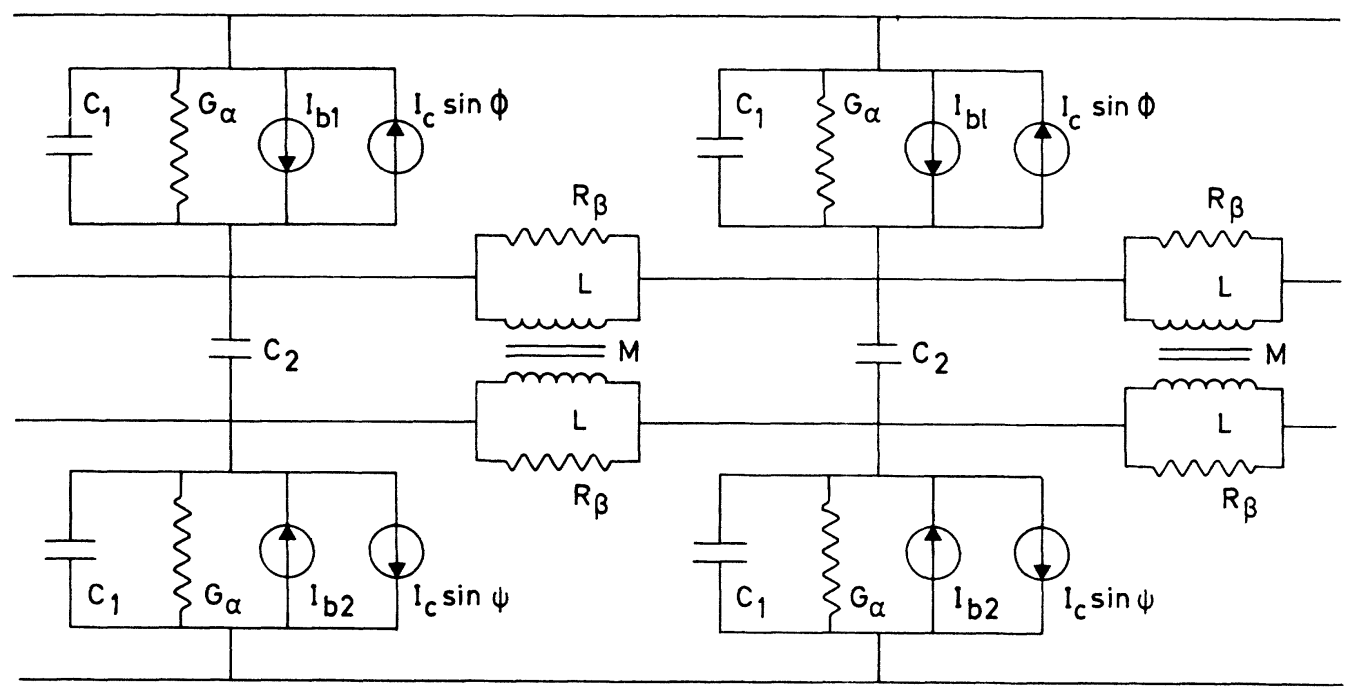

FIG. 1. Discrete electronic circuit of the system considered. The weak interaction is given by the mutual inductance $M$ and the capacitance $C_{2}$.

The equations of motion for the phase differences are, in the continuous limit, found to be

$$
\begin{aligned}
& \phi_{x x}-\phi_{t t}-\sin \phi=\alpha \phi_{t}-\beta \phi_{x x t}-\eta_{1}+\Delta_{1} \psi_{x x}+\Delta_{2} \psi_{t t}, \\
& \psi_{x x}-\psi_{t t}-\sin \psi=\alpha \psi_{t}-\beta \psi_{x x t}-\eta_{2}+\Delta_{1} \phi_{x x}+\Delta_{2} \phi_{t t} .
\end{aligned}
$$

Here $x$ and $t$ are normalized to

$$
\lambda_{J}=\left(\frac{\hbar}{2 e L I_{c}\left[1-(M / L)^{2}\right]}\right)^{1 / 2}
$$

and

$$
\omega_{0}^{-1}=\left[\frac{\hbar C_{1}}{2 e I_{c}}\left[1+\frac{C_{2}}{C_{1}}\right]\right]^{1 / 2}
$$

respectively. The normalized loss parameters $\alpha$ and $\beta$ are given by

$$
\alpha=G_{\alpha}\left(\frac{\hbar}{2 e I_{c} C_{1}\left(1+C_{2} / C_{1}\right)}\right)^{1 / 2}
$$

and

$$
\beta=\frac{L}{R_{\beta}}\left[1-\left(\frac{M}{L}\right)^{2}\right]\left(\frac{2 e I_{c}}{\hbar C_{1}\left(1+C_{2} / C_{1}\right)}\right)^{1 / 2} .
$$

The normalized bias current densities are $\eta_{1}=I_{b 1} / I_{c}$ and $\eta_{2}=I_{b 2} / I_{c}$. Finally, we have the coupling parameters as $\Delta_{1}=-M / L$ and $\Delta_{2}=-C_{2} /\left(C_{1}+C_{2}\right)$.

Note that the well-known Lorentz invariance of the unperturbed sine-Gordon equation [left-hand side of Eq. (1)] is preserved in the coupled equations, Eq. (5), for $\alpha=\beta=0$ if $\Delta_{2}=-\Delta_{1}$.

The energy of the system Eq. (5) is clearly given by expression Eq. (4) and the time rate of change in energy is then found to be

$$
\frac{d}{d t} H=\int_{-\infty}^{\infty}\left[\eta_{1} \phi_{t}+\eta_{2} \psi_{t}-\alpha\left(\phi_{t}^{2}+\psi_{t}^{2}\right)-\beta\left(\phi_{x t}^{2}+\psi_{x t}^{2}\right)\right] d x
$$

where we have assumed $\phi_{x}, \phi_{t}, \psi_{x}, \psi_{t} \rightarrow 0,|x| \rightarrow \infty$. Note that since the coupling mechanisms are nondissipative, the parameters $\Delta_{1}$ and $\Delta_{2}$ do not enter the power expression Eq. (6).

\section{PERTURBATION THEORY}

The fundamental kink solution, considered in this paper, is given by

$$
\phi^{0}=4 \tan ^{-1} Z_{ \pm}, \quad Z_{ \pm}=\exp \left( \pm \frac{x-u t}{\left(1-u^{2}\right)^{1 / 2}}\right),
$$

which is an exact solution to the left-hand side of Eq. (1), $u$ being the kink velocity and

$$
\gamma_{0}(u)=\left(1-u^{2}\right)^{-1 / 2}
$$

is the inverse Lorentz contraction of the solution. The difference between kinks and antikinks (solitons and antisolitons) is shown by the choice of sign in $Z_{ \pm}$. The solution Eq. (7) is used to describe kinks in both sineGordon systems.

One should note that in case of $\phi=\psi$ and $\alpha=\beta=\eta_{1}=\eta_{2}=0$, Eq. (5) has the exact solution:

$$
\begin{aligned}
\phi_{0}=4 \tan ^{-1}\left\{\exp \left[\gamma_{0}(u)(\xi-u \tau)\right]\right\}, \\
\xi=\frac{x}{\sqrt{1-\Delta_{1}}}, \quad \tau=\frac{t}{\sqrt{1+\Delta_{2}}} .
\end{aligned}
$$

The simplest interaction between two kinks belonging to different systems is the internal oscillation in a bunched state. Assuming $\alpha=\beta=\eta_{1}=\eta_{2}=0$ in Eq. (5) we use the kink wave forms given by

$$
\begin{aligned}
& \phi=\phi_{0}+\mu, \quad|\mu| \ll 1, \\
& \psi=\phi_{0}+\nu, \quad|\nu| \ll 1, \quad u=0 .
\end{aligned}
$$

Inserting Eq. (9) in Eq. (5) and linearizing the equations in $v$ and $\mu$, we find the equation: 
$\frac{1+\Delta_{1}}{1-\Delta_{1}}(\mu-v)_{\xi \xi}-\frac{1-\Delta_{2}}{1+\Delta_{2}}(\mu-v)_{\tau \tau}=(\mu-v) \cos \phi_{0}$.

Looking for harmonic oscillating solutions for $f(\xi, \tau)=\mu-v$ we use $\partial^{2} / \partial \tau^{2} \rightarrow-\omega^{2}$ and obtain the eigenvalue problem:

$$
\frac{1+\Delta_{1}}{1-\Delta_{1}} \frac{1+\Delta_{2}}{1-\Delta_{2}} f_{\xi \xi}-\frac{1+\Delta_{2}}{1-\Delta_{2}} \cos \phi_{0} f=-\omega^{2} f .
$$

For $\Delta_{1}=\Delta_{2}=0$ a solution to Eq. (10) is the translation mode: $\omega=0, f_{0}=2 \sin \left(\phi_{0} / 2\right)$ (see, e.g., Ref. 8). Regarding $\Delta_{1}$ and $\Delta_{2}$ as small perturbations we find by use of quantum-mechanical-like perturbation theory the eigenvalue $\omega^{2}$ for the coupled system (to first order in $\Delta_{1}$ and $\Delta_{2}$ ) to be given by

$$
\begin{gathered}
\frac{1+\Delta_{1}}{1-\Delta_{1}} \frac{1+\Delta_{2}}{1-\Delta_{2}} \int_{-\infty}^{\infty} f_{0} f_{0, \xi \xi} d \xi-\frac{1+\Delta_{2}}{1-\Delta_{2}} \int_{-\infty}^{\infty} f_{0} f_{0} \cos \phi_{0} d \xi \\
=-\int_{-\infty}^{\infty} f_{0} f_{0} d \xi \\
=\omega^{2}=\frac{2}{3} \frac{\Delta_{1}}{1-\Delta_{1}} \frac{1+\Delta_{2}}{1-\Delta_{2}} \approx \frac{2}{3} \Delta_{1}
\end{gathered}
$$

From Eq. (11) we see that the capacitance coupling $\Delta_{2}$ plays a minor role in the internal oscillation of a bunched state when $u=0$.

Turning to the adiabatic perturbation method ${ }^{5}$ we neglect the perturbation of the kink wave form arising from the right-hand sides of Eq. (5). By use of the kink solutions of the form Eq. (7) we find the interaction energy $H_{I}$ between the two weakly coupled sine-Gordon systems. Let $r$ be the distance between the two solitons. The considered kink profiles are then given by

$$
\begin{aligned}
& \phi=4 \tan ^{-1}\left\{\exp \left[\gamma_{0}\left(u_{1}\right)\left[x-\frac{r}{2}-u_{1} t\right]\right]\right\}+\sin ^{-1} \eta_{1}, \\
& \psi=4 \tan ^{-1}\left\{\exp \left[\gamma_{0}\left(u_{2}\right)\left[x+\frac{r}{2}-u_{2} t\right]\right]\right\}+\sin ^{-1} \eta_{2} .
\end{aligned}
$$

For $\left|u_{1}\right|=\left|u_{2}\right|=|u|$ we find that

$$
H_{I}=-8 \gamma_{0}(u)\left(\Delta_{1}-u_{1} u_{2} \Delta_{2}\right) \frac{r \gamma_{0}(u)}{\sinh \left[r \gamma_{0}(u)\right]} .
$$

Using Eq. (13) as the only interaction seems to be a rather crude approximation, since we have seen from Eq. (8) that the coupling parameters are first-order perturbations to the kink wave forms. However, for $u=0$ we find that $H_{0}$ [Eq. (4)] is unaffected to first order in the coupling parameters $\Delta_{1}$ and $\Delta_{2}$ when the solutions of the form $\phi_{0}$ [Eq. (8)] is used. Thus in the framework of the adiabatic perturbation method, we regard the right-hand side of Eq. (5) as small perturbations and use the unperturbed kink Eq. (7) [Eq. (12)] as a valid profile in the limit $|u| \rightarrow 0, \eta_{i}, \alpha, \beta \rightarrow 0$. As we shall see relatively good agreement between the results obtained from Eq. (13) and numerical simulation of Eq. (5) is found when the stability of the bunched state is studied.

Defining the momentum $P$ of a sine-Gordon system as

$$
P=-\int_{-\infty}^{\infty} \phi_{x} \phi_{t} d x,
$$

the result when the wave form Eq. (7) is inserted gives

$$
P=8 u \gamma_{0}(u) \text {. }
$$

The equations of motion for the two interacting kinks can then be written as

$$
\begin{aligned}
& \frac{\partial P_{1}}{\partial t}=-\alpha P_{1}-\frac{\beta}{3} P_{1}\left(1+\frac{1}{64} P_{1}^{2}\right)+2 \pi \eta_{1}-\frac{\partial H_{1}}{\partial r}, \\
& \frac{\partial P_{2}}{\partial t}=-\alpha P_{2}-\frac{\beta}{3} P_{2}\left(1+\frac{1}{64} P_{2}^{2}\right)+2 \pi \eta_{2}+\frac{\partial H_{I}}{\partial r},
\end{aligned}
$$

where

$$
\begin{aligned}
\frac{\partial H_{I}}{\partial r}= & -8 \gamma_{0}^{2}(u)\left(\Delta_{1}-u_{1} u_{2} \Delta_{2}\right) \\
& \times \frac{\sinh \left[r \gamma_{0}(u)\right]-r \gamma_{0}(u) \cosh \left[r \gamma_{0}(u)\right]}{\sinh ^{2}\left(r \gamma_{0}(u)\right)} .
\end{aligned}
$$

By setting $\alpha=\beta=\eta_{1}=\eta_{2}=0, u_{1}=-u_{2}=u,\left(|u|^{2}<<1\right)$, and $u=\frac{1}{2} \cdot \partial r / \partial t$ we find for the harmonic internal oscillation $(r<<1)$ the frequency given by

$$
\omega^{2}=\frac{2}{3} \Delta_{1} \text {. }
$$

This is consistent with the expression Eq. (11) to first order in the coupling parameters. The independence of $\Delta_{2}$ is caused by the assumption of nonrelativistic behavior $\left(|u|^{2} \ll<1\right)$, which clearly eliminates the $\Delta_{2}$ influence in the interaction Eq. (17). Note that the frequencies Eq. (18) and Eq. (11) are given for soliton-soliton interaction. If one junction sustains a soliton and the other an antisoliton, a positive $\Delta_{1}$ would give rise to a repulsive interaction. However, replacing $\Delta_{1}$ by $-\Delta_{1}$ would again give the preceding value of internal frequency in a bunched state of a soliton antisoliton pair. Now we consider the case where the two solitons move with equal velocity in a steady-state motion. By adding and subtracting Eq. (16), we get

$$
\begin{aligned}
& 0=-\alpha P-\frac{\beta}{3} P\left(1+\frac{1}{64} P^{2}\right)+\pi\left(\eta_{1}+\eta_{2}\right), \\
& 0=\pi\left(\eta_{1}-\eta_{2}\right)-\frac{\partial H_{I}}{\partial r},
\end{aligned}
$$

where $P_{1}=P_{2}=P$ in a bunched state. The first of these equations is simply the power balance velocity of the bunched state given by the average of the two bias values-i.e., (for $\beta=0$ )

$$
u=\frac{1}{\left\{1+\left[8 \alpha / \pi\left(\eta_{1}+\eta_{2}\right)\right]^{2}\right\}^{1 / 2}} .
$$

This result is of course also found (for $\beta=0$ ) when Eq. (6) is set to zero and Eq. (12) is used as kink profiles. The second equation in Eq. (19) gives the balance between the bias difference $\left(\eta_{1}-\eta_{2}\right)$ of the two sine-Gordon systems and the internal force between the kinks. This balance can only be obtained when the steady-state distance $r$ is no greater than a certain value $r_{0}$ given by the $r$ value that optimizes the force Eq. (17). Numerically this is 
found to be $r_{0} \approx 1.606\left(1-u^{2}\right)^{1 / 2}$ and the corresponding maximum internal force is

$$
\left|\frac{\partial H_{I}}{\partial r}\right|_{r=r_{0}} \approx 8 \gamma_{0}^{2}(u)\left(\Delta_{1}-u_{1} u_{2} \Delta_{2}\right) 0.310 .
$$

The criteria for the existence of a bunched state is then

$$
\frac{1}{2}\left|\eta_{1}-\eta_{2}\right|<\frac{4 \times 0.310}{\pi} \gamma_{0}^{2}(u)\left(\Delta_{1}-u^{2} \Delta_{2}\right),
$$

and when the power balance velocity (for $\beta=0$ ) Eq. (20) is inserted, this gives

$$
\begin{aligned}
\frac{1}{2}\left|\eta_{1}-\eta_{2}\right|< & \frac{4 \times 0.310}{\pi}\left[1+\left(\frac{\pi\left(\eta_{1}+\eta_{2}\right)}{8 \alpha}\right)^{2}\right] \\
& \times\left(\Delta_{1}-\frac{\Delta_{2}}{1+\left\{8 \alpha /\left[\pi\left(\eta_{1}+\eta_{2}\right)\right]\right\}^{2}}\right) .
\end{aligned}
$$

This is the threshold condition for traveling wave solutions of bunched states of solitons belonging to two weakly coupled sine-Gordon systems. When the coupling parameters satisfies the Lorentz invariant combination, $\Delta_{1}=-\Delta_{2}=\Delta$, we find that the threshold for $\beta=0$ is given by

$$
\begin{array}{r}
\frac{1}{2}\left|\eta_{1}-\eta_{2}\right|<\frac{4 \times 0.310}{\pi} \Delta\left[1+2\left[\frac{\pi\left(\eta_{1}+\eta_{2}\right)}{8 \alpha}\right)^{2}\right], \\
\Delta_{1}=-\Delta_{2}=\Delta .
\end{array}
$$

The very simple perturbation result Eq. (22) is of course valid only in the limit of small perturbations-i.e., when the right-hand sides in Eq. (5) are small. Hence it must be expected to deviate considerably from full numerical solution of Eq. (5) as we increase the bias values $\eta_{l}$. A threshold condition in the limit of high bias values is found when we note that the condition $\left|\eta_{i}\right|<1$ must be satisfied to maintain a stable kink solution in a perturbed sine-Gordon system. Thus we have

$$
\frac{1}{2}\left|\eta_{1}-\eta_{2}\right|<1-\frac{1}{2}\left(\eta_{1}+\eta_{2}\right) \text {. }
$$

The limits of Eq. (25) and Eq. (22) are then to be combined to estimate the threshold value for the bias difference in a bunched state of solitons.

\section{DISCUSSION}

Combining Eq. (25) and Eq. (23) we find good agreement with the full numerical solution of Eq. (5) in case of $\Delta_{2}=0$ and $\beta=0$. The choice of $\Delta_{2}=0$ has been made to match the study of phase locking between two parallel long Josephson junctions discussed in Ref. 3 . The numerical scheme, used to solve Eq. (5), is an explicit secondorder finite difference method where periodic boundary conditions have been used to simulate an infinite spatial dimension. Space and time grid sizes as well as the length of the system have been chosen depending on the bias values. As an initial condition we used the unperturbed soliton solutions Eq. (12) with $r=0$, and to eliminate the excess energy coming from the initial soliton wave forms we used a relatively large damping parameter $\alpha$. The results of the simulations of Eq. (5) are displayed in Fig. 2 as error bars, where the lower end of a bar represents a simulation where the kinks formed a bunched state, and the upper end of a bar represents a simulation where the kinks could not form bunched state. The perturbation result is shown as solid curves. The deviation between the results of numerical experiments and the parabolic perturbation curve is, as noted above, due to the lack of validity of the kink profiles when the perturbations are large. In particular, the Lorentz contraction of the kinks Eq. (12) is not a good approximation for high bias values. Numerical experiments show that high bias values do not give rise to the narrow Lorentz contracted kinks. Hence the coupling, which is proportional to the second derivative of the profile, is in the perturbation treatment considered too high. This is in good agreement with the comparisons made in Fig. 2. Another characteristic that can be important to the existence of a bunched state is that the individual momenta of the kinks in a bunched state are not equal when the bias values are not equal. The numerical simulations showed that the "low bias" kink has larger momentum than the "high
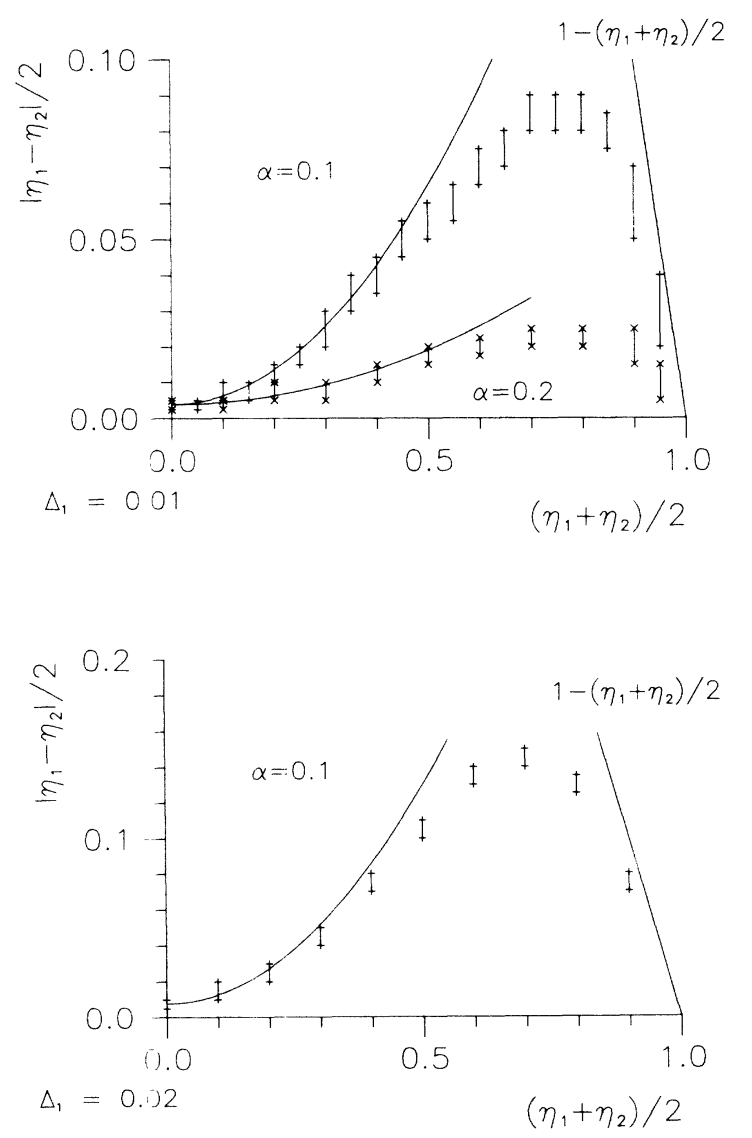

FIG. 2. The upper limit of the bias difference $\left|\eta_{1}-\eta_{2}\right| / 2$ for a bunched state of two solitons vs the average bias $\left(\eta_{1}+\eta_{2}\right) / 2$. Solid lines are perturbation results and the error bars represent the results of the numerical experiments. Only $\Delta_{2}=0$ and $\beta=0$ is considered. (a) $\Delta_{1}=0.01$. (b) $\Delta_{1}=0.02$. 
bias" kink. This is not considered in the perturbation, where the momentum is uniquely determined by the velocity Eq. (15).

We have discussed some energy conserving coupling mechanisms between sine-Gordon systems. Criteria for existence of a bunched state of kinks from different weakly coupled systems have been obtained by simple perturbation arguments. Comparisons with numerical simulations of the coupled partial differential equations, Eq. (5), and perturbation treatment have shown good agreement in the expected region of parameters, when the stability of the bunched states are studied. Also, we have shown consistency between two different perturbation methods leading to the frequency of the internal oscillation of a bunched state [Eqs. (11) and (18)].
We close this paper by noting that the threshold condition for the existence of bunched states can be closely related to the locking range in phase locking experiments recently obtained with two long inductively coupled Josephson junctions. ${ }^{3}$ Our results in this paper are obtained for infinite junction length, and consequently, the boundary reflections of the magnetic fluxons are not considered. However, if the junctions are made very long, or annular, the boundary effect would vanish.

\section{ACKNOWLEDGMENTS}

One of the authors (N.G.J.) is grateful to the Otto Mønsteds fond and to the Christian og Ottilia Brorsons rejselegat for financial support.
${ }^{1}$ Y. S. Kivshar and B. A. Malomed, Phys. Rev. B 37, 9325 (1988).

${ }^{2}$ O. M. Braun, Y. S. Kivshar, and A. M. Kosevich, J. Phys. C 21, 3881 (1988).

${ }^{3}$ T. Holst, J. B. Hansen, N. Grønbech-Jensen, and J. A. Blackburn, Phys. Rev. B 42, 127 (1990).

${ }^{4}$ N. Grønbech-Jensen, R. D. Parmentier, and N. F. Pedersen, Phys. Lett. A 142, 427 (1989).
${ }^{5}$ D. W. McLaughlin and A. C. Scott, Phys. Rev. A 18, 1652 (1978).

${ }^{6}$ N. F. Pedersen, M. R. Samuelsen, and D. Welner, Phys. Rev. B 30, 4057 (1984)

${ }^{7}$ O. A. Levring, N. F. Pedersen, and M. R. Samuelsen, J. Appl. Phys. 54, 987 (1983).

${ }^{8}$ J. Rubinstein, J. Math. Phys. 11, 258 (1970). 